PROCEEDINGS OF THE

AMERICAN MATHEMATICAL SOCIETY

Volume 131, Number 10, Pages 3257-3265

S 0002-9939(02)06840-5

Article electronically published on December 30, 2002

\title{
PSEUDOCOMPACT WHYBURN SPACES NEED NOT BE FRÉCHET
}

\author{
JAN PELANT, MIHAIL G. TKACHENKO, VLADIMIR V. TKACHUK, \\ AND RICHARD G. WILSON \\ (Communicated by Alan Dow)
}

\begin{abstract}
We prove in ZFC that there exists a Tychonoff pseudocompact scattered AP-space of uncountable tightness. We give some sufficient and necessary conditions for a $\mathcal{P}$-space to be AP as well as a characterization of AP-property in linearly ordered topological spaces.
\end{abstract}

\section{INTRODUCTION}

If $X$ is a topological space and $F \subset X$, let us say that $F$ is almost closed if $\bar{F} \backslash F$ is a one-point set. If $\bar{F} \backslash F=\{x\}$, we denote it by $F \rightarrow x$. It is natural to say that the topology of a space $X$ is determined by almost closed subspaces if, for any nonclosed $A \subset X$ and any $x \in \bar{A} \backslash A$, there is an almost closed $F \subset A$ such that $F \rightarrow x$. By analogy with the concepts of Fréchet-Urysohn and sequential spaces, we say that a topological space $X$ is weakly determined by almost closed subspaces if, for any non-closed $A \subset X$, there is an almost closed $B \subset A$ such that $B \rightarrow x \notin A$.

The spaces determined by almost closed subspaces were first introduced by Whyburn [Wh] who baptized them accessibility spaces and studied the properties of pseudo-open maps onto accessibility spaces. Later Okromeshko [Ok] obtained more results in the same direction. Twenty-three years later this concept appeared in the paper of Pultr and Tozzi $[\mathrm{PT}]$ in the context of categorical topology. They called the relevant class "AP-spaces", AP standing for "Approximation-by-Points". When Bella [Be1] and Simon [Si] studied topological properties of AP-spaces being unaware of the papers $[\overline{\mathrm{Wh}}]$ and $[\mathrm{Ok}]$, they used the terminology of $[\mathrm{PT}]$. The spaces weakly determined by almost closed subsets were introduced in [Si] where they were called WAP-spaces, the first letter standing for "weakly". In spite of being uninformative, the names AP and WAP became entrenched and were further used in the papers $[\mathrm{Be} 2, \mathrm{BY}]$ and $[\mathrm{TY}]$.

The situation changed recently when Arhangel'skii communicated to the authors of this paper and other specialists in the field, that the concept of an AP-space was

Received by the editors November 30, 2001 and, in revised form, April 18, 2002.

2000 Mathematics Subject Classification. Primary 54A20, 54D99, 54F05, 54G10, 54G20.

Key words and phrases. Whyburn space, weakly Whyburn space, AP-space, WAP-space, pseudocompact space, countably compact space, scattered space, Lindelöf $\mathcal{P}$-space, linearly ordered space.

This research was supported by Consejo Nacional de Ciencia y Tecnología (CONACYT) of México, grant 400200-5-28411E.

The first author's research was supported by the grant GA CR 201/00/1466. 
first introduced by Whyburn in Wh. That is why the authors of this paper have decided (after consultation with other specialists) to give credit to Whyburn and call AP-spaces Whyburn spaces. Of course, WAP-spaces automatically acquire the title "weakly Whyburn spaces". As a transitional measure, we use the old terms in the Abstract and Introduction but the main text contains only new terminology.

The first to show that some AP-spaces are Fréchet was Bella Be1 who proved that any scattered compact AP-space is Fréchet-Urysohn. In BY] it was proved that "scattered" can be omitted in this result. Tkachuk and Yaschenko established that every countably compact AP-space is Fréchet-Urysohn and asked ([TY], Problem 4.7) whether every pseudocompact AP-space must also be Fréchet-Urysohn. The main result of this paper is an answer to Problem 4.7: we construct a ZFC example of a scattered pseudocompact zero-dimensional Tychonoff space which is AP but has uncountable tightness (and even uncountable o-tightness). We also study AP-property in $P$-spaces and give a characterization of the AP-property in linearly ordered spaces.

Our terminology is standard and follows [En]. All spaces are assumed to be Hausdorff. Given two infinite sets $A$ and $B$ we say that $A \subset^{*} B$ if $A \backslash B$ is finite. Analogously, if $f, g \in \omega^{\omega}$, then $f<^{*} g$ if there is $k \in \omega$ such that $f(n)<g(n)$ for all $n \geq k$. A set $F \subset \omega^{\omega}$ is bounded if there is a $g \in \omega^{\omega}$ such that $f<^{*} g$ for every $f \in F$. The symbol $\mathfrak{b}$ denotes the minimal cardinality of unbounded subsets of $\omega^{\omega}$. A family $D \subset \omega^{\omega}$ is dominating if, for any $f \in \omega^{\omega}$ there is $g \in D$ such that $f<^{*} g$. The symbol $\mathfrak{d}$ stands for the minimal cardinality of dominating subsets of $\omega^{\omega}$. It is clear that $\mathfrak{b} \leq \mathfrak{d}$ and the equality $\mathfrak{b}=\mathfrak{d}$ follows from $\mathrm{CH}$ as well as from MA [vD, so the statement $\mathfrak{b}=\mathfrak{d}$ is compatible with the usual axioms of ZFC.

We say that $X$ is a $P$-space if any $G_{\delta}$-subset of $X$ is open. The $\omega$-modification of a space $(X, \tau)$ is a space with the underlying set $X$ whose topology is generated by $G_{\delta}$-subsets of $(X, \tau)$. A set $A \subset X$ is Whyburn closed if $\bar{F} \subset A$ for any almost closed $F \subset A$. If we want to emphasize that a set $F$ is almost closed in a space $X$, we say that $F$ is $X$-almost closed or almost closed in $X$.

\section{The Whyburn property in Lindelöf and pSeudocompact spaces}

We give examples of pseudocompact Whyburn spaces of uncountable tightness giving a complete answer to Problem 4.7 from TY]. We also consider the Whyburn property in $P$-spaces and give its characterization in linearly ordered spaces.

2.1. Theorem. If the equality $\mathfrak{b}=\mathfrak{d}$ holds, then there exists a Tychonoff scattered separable pseudocompact Whyburn space of uncountable tightness.

Proof. Take a dominating family $\Phi=\left\{\varphi_{\alpha}: \alpha<\mathfrak{b}\right\}$ in $\left(\omega^{\omega},<^{*}\right)$. Since $\mathfrak{b}=\mathfrak{d}$, we can suppose that $\Phi$ is well-ordered by $<^{*}$ so that $\alpha \rightarrow \varphi_{\alpha}$ is an order isomorphism and, in particular, $\varphi_{\alpha}<^{*} \varphi_{\alpha+1}$ for each $\alpha<\mathfrak{b}$. For the set $C=\omega \times \omega$, let $C_{n}=\{n\} \times \omega$ for each $n \in \omega$. Given $\varphi_{\alpha} \in \Phi$, we define the sets $D_{\alpha}=\left\{(m, n) \in C: n \leq \varphi_{\alpha}(m)\right\}$ and $U_{\alpha}=C \backslash D_{\alpha}$. Call a set $S \subset C$ a selector if $\left|S \cap C_{n}\right| \leq 1$ for any $n \in \omega$. By transfinite induction on $\alpha<\mathfrak{b}$ we define an almost disjoint family $\mathcal{A}$ on the set $C$ with the following properties:

(i) $\mathcal{A}=\bigcup\left\{\mathcal{A}_{\alpha}: \alpha<\mathfrak{b}\right\}$ and $\mathcal{A}$ consists of selectors on $C$;

(ii) the family $\mathcal{A} \cup\left\{C_{n}: n \in \omega\right\}$ is maximal almost disjoint;

(iii) $A \subset D_{\alpha}$ and $A \subset^{*} U_{\beta}$ whenever $\beta<\alpha<\mathfrak{b}$ and $A \in \mathcal{A}_{\alpha}$. 
For $\alpha=0$, it is possible to choose a maximal almost disjoint family $\mathcal{A}_{0}$ of selectors contained in $D_{0}$. Now assume that we have defined the families $\mathcal{A}_{\beta}$ for all $\beta<\alpha$, where $\alpha<\mathfrak{b}$. If $\alpha=\delta+1$, then, since the set $D_{\alpha} \cap U_{\delta}$ is infinite, we can choose a maximal almost disjoint family $\mathcal{A}_{\alpha}$ of selectors contained in $D_{\alpha} \cap U_{\delta}$.

If $\alpha=\lim \alpha$, then choose any family $\mathcal{A}_{\alpha}$ of selectors such that

(iv) every element of $\mathcal{A}_{\alpha}$ is a subset of $D_{\alpha}$;

(v) $\mathcal{A}_{\alpha}$ is almost disjoint and the set $A \cap D_{\beta}$ is finite for any $\beta<\alpha$ and $A \in \mathcal{A}_{\alpha}$;

(vi) $\mathcal{A}_{\alpha}$ is maximal with respect to the properties (iv) and (v).

We claim that the family $\mathcal{A}=\bigcup\left\{\mathcal{A}_{\alpha}: \alpha<\mathfrak{b}\right\}$ satisfies (i), (ii) and (iii). Let us check first that $\mathcal{A} \cup\left\{C_{n}: n \in \omega\right\}$ is almost disjoint. If $A, B \in \mathcal{A}$ are distinct sets, then $A \in \mathcal{A}_{\alpha}$ and $B \in \mathcal{A}_{\beta}$; assume without loss of generality that $\alpha \leq \beta$. If $\alpha<\beta$, then $A \subset D_{\alpha}$ and $B \cap D_{\alpha}$ is finite so the set $A \cap B$ is finite. If $\alpha=\beta$, then $A \cap B$ is finite because $\mathcal{A}_{\alpha}$ is almost disjoint. Since $\mathcal{A}$ consists of selectors, the family $\mathcal{A} \cup\left\{C_{n}: n \in \omega\right\}$ is also almost disjoint.

To see that $\mathcal{A}^{\prime}=\mathcal{A} \cup\left\{C_{n}: n \in \omega\right\}$ is maximal, take any infinite $D \subset C$ such that $D \cap A$ is finite for any $A \in \mathcal{A}^{\prime}$. Since $D \cap C_{n}$ is finite for each $n$, the set $D$ contains an infinite selector $D^{\prime}$; we assume, without loss of generality, that $D=D^{\prime}$, i.e., $D$ is a selector. Since the family $\Phi$ dominates, we have $D \subset^{*} D_{\gamma}$ for some $\gamma<\mathfrak{b}$ and hence the set $D \cap D_{\gamma}$ is infinite. Denote by $\alpha$ the minimal element of the set $\left\{\delta<\mathfrak{b}: D \cap D_{\delta}\right.$ is infinite $\}$. If $\alpha=\eta+1$, then some infinite $E \subset D$ lies in $D_{\alpha} \cap U_{\eta}$. Since $E$ is a selector and $\mathcal{A}_{\alpha}$ is a maximal family of selectors on $D_{\alpha} \cap U_{\eta}$, the set $E$ has an infinite intersection with some $A \in \mathcal{A}_{\alpha}$ which is a contradiction. If $\alpha$ is a limit ordinal, then $H \subset D_{\alpha}$ for some infinite $H \subset D$. By definition of $\alpha$ the set $H \cap D_{\beta}$ is finite for each $\beta<\alpha$ and therefore $H$ is a selector on $D_{\alpha}$ which satisfies properties (iv) and (v) for the elements of $\mathcal{A}_{\alpha}$. Since $\mathcal{A}_{\alpha}$ is a maximal family of selectors with (iv) and (v) the set $H \cap A$ is infinite for some $A \in \mathcal{A}_{\alpha}$ which gives the final contradiction.

For each $A \in \mathcal{A}$ we take any point $p_{A} \notin C$ and a point $p \notin C \cup\left\{p_{A}: A \in \mathcal{A}\right\}$; then $X=\{p\} \cup\left\{p_{A}: A \in \mathcal{A}\right\} \cup C$ is the underlying set of a space we need to prove our theorem. All points of $C$ are isolated in $X$ and the neighbourhood base of any $p_{A}$ is the family $\left\{\left\{p_{A}\right\} \cup(A \backslash F): F\right.$ is a finite subset of $\left.A\right\}$. Finally, a base at $p$ is formed by the sets $O_{\alpha}=\{p\} \cup U_{\alpha} \cup\left\{p_{A}: A \in \bigcup_{\beta>\alpha} \mathcal{A}_{\beta}\right\}$, where $\alpha<\mathfrak{b}$.

It is immediate that $X$ is a scattered zero-dimensional Tychonoff space. Observe that $X$ is separable because $C$ is dense in $X$. Note also that, for any $A \subset C$, we have $p \in \bar{A}$ if and only if $A \cap C_{n}$ is infinite for some $n \in \omega$. Now it is easy to show that $X$ is pseudocompact. It suffices to verify that any infinite $D \subset C$ has an accumulation point in $X$. Since the family $\mathcal{A}^{\prime}=\mathcal{A} \cup\left\{C_{n}: n \in \omega\right\}$ is maximal, the set $A \cap D$ is infinite for some $A \in \mathcal{A}^{\prime}$. If $A=C_{n}$ for some $n$, then $p \in \bar{D} \backslash D$. If not, then $A \in \mathcal{A}$ and hence $p_{A}$ is the respective accumulation point for $D$ which proves that $X$ is pseudocompact.

To see that $X$ is Whyburn take any $D \subset X$ and any $x \in \bar{D} \backslash D$. Clearly, $x \in\{p\} \cup\left\{p_{A}: A \in \mathcal{A}\right\}$. If $x=p_{A}$ for some $A \in \mathcal{A}$, then $D \cap A$ is a sequence in $D$ which converges to $x$. If $x=p$ and $x \in \overline{D \cap C}$, then $S=D \cap C_{n}$ is infinite for some $n$ and hence $S$ is a sequence in $D$ which converges to $x$. If $x \notin \overline{D \cap C}$, then $x \in \overline{D \cap P}$ where $P=\left\{p_{A}: A \in \mathcal{A}\right\}$. As a consequence $B=D \cap P$ is an almost closed subset of $D$ with $B \rightarrow x$ so the Whyburn property of $X$ is established. Observe finally that $X$ has uncountable tightness because $p \in \bar{P}$ but $p \notin \bar{D}$ for any countable $D \subset P$. 
To prove the next theorem, we need an auxiliary construction to be carried out for an arbitrary countably infinite set $D$ which will be identified with the discrete space whose underlying set is $D$. The letter $K$ stands for the Cantor set; the mappings $\pi_{D}: D \times K \rightarrow D$ and $\pi_{K}: D \times K \rightarrow K$ are the respective natural projections. If $A \subset D \times K$, then $A(d)$ denotes the set $\pi_{K}(A \cap(\{d\} \times K))$ for any $d \in D$. A set $A \subset D \times K$ is called admissible if it has the following properties:

(i) the set $i(A)=\pi_{D}(A)$ is infinite;

(ii) the set $A(d)$ is clopen in $K$ for every $d \in i(A)$ and the family $\{A(d): d \in$ $i(A)\}$ is disjoint.

A family $\mathcal{A}$ of admissible subsets of $D \times K$ is called essentially disjoint if, for any distinct $A, B \in \mathcal{A}$, the set $\{d \in D: A(d) \cap B(d) \neq \emptyset\}$ is finite. Denote by $\mathcal{A}(D)$ any maximal essentially disjoint family of admissible subsets of $D \times K$. Assign a point $p_{A}$ to any set $A \in \mathcal{A}(D)$.

We can now define our auxiliary space $P(D)=(D \times K) \cup\left\{p_{A}: A \in \mathcal{A}(D)\right\}$. The local bases at the points of $D \times K$ are given by their usual local bases in $D \times K$ (remember that $D$ is endowed with the discrete topology). Given an $A \in \mathcal{A}(D)$, a local base at the point $p_{A}$ is the family $\left\{\left\{p_{A}\right\} \cup(A \backslash(F \times K)): F \subset D\right.$ is finite $\}$.

2.2. Lemma. The space $P(D)$ is Tychonoff, Fréchet-Urysohn, pseudocompact and zero-dimensional; $D \times K$ is an open subspace of $P(D)$ and the set $\left\{p_{A}: A \in \mathcal{A}(D)\right\}$ is closed and discrete.

Proof. The space $P(D)$ is Hausdorff because $\mathcal{A}(D)$ is essentially disjoint; it is zerodimensional because $U \cap(D \times K)$ is clopen in $D \times K$ for any basic neighbourhood $U$ of any point $p_{A}$. The proof of all other properties except for pseudocompactness is easy so we will only check that $P(D)$ is pseudocompact. It suffices to show that, for any infinite family $\mathcal{U}$ of non-empty clopen subsets of $D \times K$, there is a point $x \in P(D)$ such that every neighbourhood of $x$ meets infinitely many elements of $\mathcal{U}$.

If the family $\mathcal{U}_{d}=\{U \in \mathcal{U}: U(d) \neq \emptyset\}$ is infinite for some $d \in D$, then such a point exists because the space $K$ is compact. If every $\mathcal{U}_{d}$ is finite, then the set $\{d \in D: U(d) \neq \emptyset$ for some $U \in \mathcal{U}\}$ is infinite and hence we can find an admissible set $B \subset D \times K$ and an injection $d \rightarrow U_{d}$ of the set $i(B)$ into $\mathcal{U}$ such that $B(d) \subset U_{d}(d)$ for each $d \in i(B)$. The family $\mathcal{A}(D)$ being maximal essentially disjoint, we can find $A \in \mathcal{A}(D)$ such that $A(d) \cap B(d) \neq \emptyset$ for infinitely many $d$ 's. To finish our proof observe that each neighbourhood of the point $p_{A}$ intersects infinitely many elements of $\mathcal{U}$.

2.3. Theorem. There is (in ZFC) a Tychonoff pseudocompact (non-separable) scattered Whyburn space of uncountable o-tightness.

Proof. Denote by $L$ the subset of $\mathfrak{c}^{+}$which consists of limit ordinals of countable cofinality. Considering $L$ with its order topology it is natural to say that a countably infinite set $S \subset L$ is a sequence converging to $\alpha \in L$ if $S \subset \alpha, \sup S=\alpha$ and $S \cap \beta$ is finite for any $\beta<\alpha$.

If $\alpha \in L$ and $\alpha=\beta+\omega$ for some $\beta<\mathfrak{c}^{+}$(i.e., if $\alpha$ is an isolated point of $L$ ) let $\mathcal{D}_{\alpha}=\{[\beta, \alpha)\}$, where $[\beta, \alpha)=\{\gamma: \beta \leq \gamma<\alpha\}$. If $\alpha$ is not isolated in $L$, define $\mathcal{D}_{\alpha}$ to be any maximal almost disjoint family of sequences converging to $\alpha$. It is a routine to prove that $\mathcal{D}=\bigcup\left\{\mathcal{D}_{\alpha}: \alpha \in L\right\}$ is a maximal almost disjoint family of countable subsets of $\mathfrak{c}^{+}$.

Apply Lemma 2.2 to fix the family $\mathcal{A}(D)$, the points $\left\{p_{A}: A \in \mathcal{A}(D)\right\}$ and the space $P(D)$ for every $D \in \mathcal{D}$. Choose a new point $p$ and define $X=\{p\} \cup\left\{p_{A}\right.$ : 
$A \in \mathcal{A}(D), D \in \mathcal{D}\} \cup\left(\mathfrak{c}^{+} \times K\right)$ to be the underlying set of the space we want to construct. Given a point $x=(\alpha, z) \in \mathfrak{c}^{+} \times K$, take a clopen local base $\left\{W_{n}: n \in \omega\right\}$ of $z$ in the space $K$ and let $U_{n}=\{\alpha\} \times W_{n}$ for each $n \in \omega$. We declare the family $\mathcal{B}_{x}=\left\{U_{n}: n \in \omega\right\}$ to be a local base of the point $x$ in $X$. Observe that the local bases thus defined generate the product topology on $\mathfrak{c}^{+} \times K$ if $\mathfrak{c}^{+}$is considered with the discrete topology.

If $A \in \mathcal{A}(D)$ for some $D \in \mathcal{D}$, then the local base $\mathcal{B}_{x}$ at the point $x=p_{A}$ is the same as in the space $P(D)$ (recall that $P(D)=D \times K \subset \mathfrak{c}^{+} \times K$ is a subset of $X$ ). Finally, declare the local base of $X$ at the point $p$ to be the family $\mathcal{B}_{p}=\left\{V_{\alpha}: \alpha \in L\right\}$ where

$$
V_{\alpha}=\{p\} \cup\left(\left\{\beta: \alpha<\beta<\mathfrak{c}^{+}\right\} \times K\right) \cup\left\{p_{A}: A \in \mathcal{A}(D), D \in \bigcup\left\{\mathcal{D}_{\beta}: \beta>\alpha\right\}\right\}
$$

for each $\alpha \in L$.

Let us prove that $X$ has all the required properties. It is immediate that $X$ is a $T_{1}$-space, so to prove that $X$ is Tychonoff it suffices to show that it is zerodimensional. We will establish a stronger fact, namely that all elements of the local bases defined above are clopen in $X$.

If $x=(\alpha, z) \in \mathfrak{c}^{+} \times K$ and $U \in \mathcal{B}_{x}$, then $W=\left(\mathfrak{c}^{+} \times K\right) \backslash U$ is open in $\mathfrak{c}^{+} \times K$ and therefore $W$ is a neighbourhood of any $y \in\left(\mathfrak{c}^{+} \times K\right) \backslash U$. Given an $A \in \mathcal{A}(D)$ for some $D \in \mathcal{D}$, it is immediate from the definition of the local base at $y=p_{A}$ that $\left\{p_{A}\right\} \cup\left(\left(\mathfrak{c}^{+} \times K\right) \backslash U\right)$ is an open neighbourhood of $y$ which does not meet $U$. Now, if $y=p$, then $V_{\beta} \cap U=\emptyset$ for any $\beta \in L, \beta>\alpha$ and this finishes the proof that $U$ is a clopen set.

Now we will show that, if $x=p_{A}$ for some $A \in \mathcal{A}(D), D \in \mathcal{D}$, then every $U \in \mathcal{B}_{x}$ is a clopen set. Observe first that $W=\left(\mathfrak{c}^{+} \times K\right) \backslash U$ is a clopen set in $\mathfrak{c}^{+} \times K$ so every point from $W$ is not in the closure of $U$. If $y=p_{B}$ is distinct from $x$, then either $B \in \mathcal{A}(D)$ or $i(A) \cap i(B)$ is finite because the family $\mathcal{D}$ is almost disjoint. Now observe that $p_{B}$ belongs to the closure of a set $T \subset \mathfrak{c}^{+} \times K$ if and only if $T$ intersects infinitely many sets of the family $\{B(d): d \in i(B)\}$. If $B \in \mathcal{A}(D)$ this is impossible for $T=A$ because the family $\mathcal{A}(D)$ is essentially disjoint and if $B \in \mathcal{A}\left(D^{\prime}\right)$ for some $D^{\prime} \neq D$, then $i(B) \cap i(A)$ is finite being contained in the finite set $D \cap D^{\prime}$. Finally, to see that $p$ is not in the closure of $U$ observe that $V_{\alpha} \cap U=\emptyset$ for any $\alpha \in L$ with $\alpha>\sup (D)$.

To establish that $V_{\alpha}$ is clopen for any $\alpha \in L$ observe that $V_{\alpha} \cap\left(\mathfrak{c}^{+} \times K\right)$ is clopen in $\mathfrak{c}^{+} \times K$ so no point of $\left(\mathfrak{c}^{+} \times K\right) \backslash V_{\alpha}$ can be in the closure of $V_{\alpha}$. If $p_{A} \notin V_{\alpha}$, then $A \in \mathcal{A}(D)$ for some $D \in \mathcal{D}_{\beta}$ with $\beta \leq \alpha$. By definition of $\mathcal{D}_{\beta}$ we have $\sup (D) \leq \alpha$ and therefore $\left\{p_{A}\right\} \cup(D \times K)$ is a neighbourhood of $p_{A}$ which misses $V_{\alpha}$. Thus we have finally proved that $X$ is a Tychonoff zero-dimensional space.

It is easy to see that $\mathcal{U}=\left\{\{\alpha\} \times K: \alpha<\mathfrak{c}^{+}\right\}$is a family of clopen subsets of $X$ such that $p \in \bar{\cup} \mathcal{U}$ but $p \notin \overline{\mathcal{U}^{\prime}}$ for any $\mathcal{U}^{\prime} \subset \mathcal{U}$ with $\left|\mathcal{U}^{\prime}\right| \leq \mathfrak{c}$. This shows that $X$ has uncountable $o$-tightness.

To establish that $X$ is pseudocompact take any discrete family $\left\{U_{n}: n \in \omega\right\}$ of non-empty open subsets of $X$. Since $\mathfrak{c}^{+} \times K$ is open and dense in $X$ we can assume that $U_{n}$ is a clopen subset of $\mathfrak{c}^{+} \times K$ for each $n \in \omega$. Since each $K_{\alpha}=\{\alpha\} \times K$ is compact, only finitely many $U_{n}$ 's can intersect each $K_{\alpha}$. Thus, choosing smaller clopen sets and passing to an appropriate infinite subfamily, we can construct a discrete family $\left\{V_{n}: n \in \omega\right\}$ of non-empty clopen subsets of the space $X$ such that, for all $m, n \in \omega$, we have $\left|i\left(V_{n}\right)\right|=1, i\left(V_{n}\right) \cap i\left(V_{m}\right)=\emptyset$ if $n \neq m$ and 
the family $\left\{\pi_{K}\left(V_{n}\right): n \in \omega\right\}$ is disjoint. By maximality of $\mathcal{D}$ there exists a set $D \in \mathcal{D}$ such that the set $D^{\prime}=D \cap\left(\bigcup\left\{i\left(V_{n}\right): n \in \omega\right\}\right)$ is infinite. It is easy to see that the set $B=\bigcup\left\{V_{n}: i\left(V_{n}\right) \subset D^{\prime}\right\}$ is admissible. By maximality of the family $\mathcal{A}(D)$ there exists $A \in \mathcal{A}(D)$ such that $A(\alpha) \cap B(\alpha) \neq \emptyset$ for infinitely many $\alpha$ 's. As a consequence, $p_{A}$ is an accumulation point of the family $\left\{V_{n}: n \in \omega\right\}$, a contradiction. Thus $X$ is pseudocompact.

Finally we show that $X$ is a Whyburn space. It is evident that all points of $X$ except for $p$ are Fréchet-Urysohn points so we must only check the Whyburn property at $p$. Let $T$ be the set $\left\{p_{A}: A \in \mathcal{A}(D), D \in \mathcal{D}\right\}$. Suppose that $p \in \bar{A} \backslash A$ for some $A \subset X$. If $p \in \overline{A \cap T}$, then $B=A \cap T$ is an almost closed subset of $A$ with $B \rightarrow p$. If not, then $p \in \overline{A \cap\left(\mathfrak{c}^{+} \times K\right)}$ and therefore the cardinality of the set $A^{\prime}=A \cap\left(\mathfrak{c}^{+} \times K\right)$ is equal to $\mathfrak{c}^{+}$. Since there are only $\mathfrak{c}$ points in $K$, there exists a $t \in K$ such that the set $B=\left\{\alpha<\mathfrak{c}^{+}:(\alpha, t) \in A^{\prime}\right\}$ has cardinality $\mathfrak{c}^{+}$. It is easy to check that $F=B \times\{t\}$ is an almost closed subset of $A$ with $F \rightarrow p$.

2.4. Remark. Any countably compact regular space of character $\leq \omega_{1}$ is semiradial under MA $+\neg \mathrm{CH}\left[\mathrm{Be} 2\right.$, Theorem $\left.5^{\prime}\right]$. Since every semiradial space is weakly Whyburn [Be1, Proposition 1], we conclude that, under $M A+\neg C H$, every regular countably compact space of character $\leq \omega_{1}$ is weakly Whyburn. In particular, the space $\{0,1\}^{\omega_{1}}$ is weakly Whyburn if MA $+\neg \mathrm{CH}$ holds. On the other hand, if $\mathrm{CH}$ is fulfilled, then the space $\beta \omega$ embeds in $\{0,1\}^{\omega_{1}}$ as a closed subspace and it is known (see $[\mathrm{TY}]$ ) that $\beta \omega$ is not weakly Whyburn. This shows that it is independent of ZFC whether $\{0,1\}^{\omega_{1}}$ is weakly Whyburn.

2.5. Theorem. Suppose that $X$ is a Whyburn Lindelöf $P$-space. Then $t(X) \leq \omega_{1}$.

Proof. Take any $A \subset X$ and any $x \in \bar{A}$. Fix an almost closed $F \subset A$ with $F \rightarrow x$. Let $\gamma$ be a maximal disjoint family of open subsets of $F$ such that $x \notin \bar{V}$ for any $V \in \gamma$. The family $\gamma$ is uncountable because $\bar{F}$ is a $P$-space. Since $x \in \bar{U} \gamma$, there is an almost closed set $G \subset \bigcup \gamma$ such that $G \rightarrow x$; clearly $G$ is also uncountable. For any $V \in \gamma$ such that $V \cap G \neq \emptyset$ choose a point $x_{V} \in V \cap G$. The set $Q=\left\{x_{V}: V \in \gamma\right.$ and $\left.V \cap G \neq \emptyset\right\}$ is discrete and again uncountable; it is easy to see that every subset of $Q$ is closed in $G$. Therefore $Q \backslash U$ is closed and discrete in $X$ for any open neighbourhood $U$ of the point $x$. Since $X$ is Lindelöf, the set $Q \backslash U$ is countable. As a consequence, if we choose $\omega_{1}$ points from $Q$ we have constructed a set $B \subset A$ with $|B| \leq \omega_{1}$ and $x \in \bar{B}$.

2.6. Example. There exists a weakly Whyburn regular Lindelöf $P$-space of arbitrarily big tightness. Thus, not every regular Lindelöf $P$-space is Whyburn.

Proof. Take any cardinal $\kappa \geq \omega$ and denote by $Y$ the ordinal $\kappa^{+}+1$ with the order topology. The $\omega$-modification $X$ of the space $Y$ is a Lindelöf $P$-space Us] and has tightness $\kappa^{+}$. Since the topology of $X$ is stronger than the order topology of $Y$, the space $X$ is scattered and hence it is weakly Whyburn [TY]. If $\kappa=\omega_{1}$, then $X$ is not Whyburn because its tightness is equal to $\omega_{2}$ while any Whyburn Lindelöf $P$-space has tightness $\leq \omega_{1}$ by Theorem 2.5 .

\subsection{Proposition. Every $P$-space $X$ of character $\leq \omega_{1}$ is Whyburn.}

Proof. Take any $A \subset X$ and any $x \in \bar{A} \backslash A$. Fix a local base $\mathcal{B}=\left\{U_{\alpha}: \alpha<\omega_{1}\right\}$ at the point $x$ such that $U_{\beta} \subset U_{\alpha}$ whenever $\alpha<\beta$. Such a choice is possible because $X$ is a $P$-space. Choose a point $x_{\alpha} \in U_{\alpha} \cap A$ for each $\alpha<\omega_{1}$. The set 
$F=\left\{x_{\alpha}: \alpha<\omega_{1}\right\} \subset A$ is almost closed because each countable subset of $X$ is closed and $F \rightarrow x$ because $\mathcal{B}$ is a local base at $x$.

2.8. Corollary. Every Lindelöf $P$-space of pseudocharacter $\leq \omega_{1}$ is Whyburn. In particular, if $X$ is a Lindelö $P$-space and $|X| \leq \omega_{1}$, then $X$ is a Whyburn space.

Proof. Recall that all spaces under consideration are assumed to be Hausdorff. A standard proof shows that every Hausdorff Lindelöf $P$-space is regular. It is also routine to prove that if a regular Lindelöf $P$-space has pseudocharacter $\leq \omega_{1}$, then its character is $\leq \omega_{1}$. Now apply Proposition 2.7.

Let $L$ be a linearly ordered topological space. We denote by $L^{*}$ the Dedekind completion of the space $L$ and by "<" the order on both $L$ and $L^{*}$. To avoid confusion as to where the intervals are taken, we denote these indicating the relevant set as a subindex. Thus, given a point $x \in L$, we have the intervals $(\leftarrow, x]_{L}=\{y \in$ $L: y \leq x\}, \quad(\leftarrow, x]_{L^{*}}=\left\{y \in L^{*}: y \leq x\right\},[x, \rightarrow)_{L}=\{y \in L: x \leq y\}$ and $[x, \rightarrow)_{L^{*}}=\left\{y \in L^{*}: x \leq y\right\}$. The intervals of other types (e.g., $(x, y)_{L},(x, \rightarrow)_{L^{*}}$, etc.) are defined analogously.

2.9. Definition. Call a linearly ordered space $L$ left incomplete at a point $x \in L$ if there is an $L^{*}$-almost closed set $F \subset\left(L^{*} \backslash L\right) \cap(\leftarrow, x)_{L^{*}}$ such that $F \rightarrow x$. Analogously, the space $L$ is right incomplete at a point $x \in L$ if there is an $L^{*}$ almost closed set $F \subset\left(L^{*} \backslash L\right) \cap(x, \rightarrow)_{L^{*}}$ such that $F \rightarrow x$. A linearly ordered space $L$ is club incomplete if, for any $x \in L$ with $\chi\left(x,(\leftarrow, x]_{L}\right)>\omega\left(\chi\left(x,[x, \rightarrow)_{L}\right)>\omega\right)$, the space $L$ is left (right) incomplete at the point $x$.

2.10. Theorem. A linearly ordered topological space $L$ is Whyburn if and only if it is club incomplete.

Proof. Suppose that $L$ is club incomplete, $A \subset L$ and $x \in \bar{A} \backslash A$. We can assume without loss of generality that $A \subset(\leftarrow, x)_{L}$ or $A \subset(x, \rightarrow)_{L}$. Since these cases are identical, we assume that $A \subset(\leftarrow, x)_{L}$. If $\chi\left(x,(\leftarrow, x]_{L}\right)=\omega$, then there is nothing to prove so suppose that $\chi\left(x,(\leftarrow, x]_{L}\right)=\kappa>\omega$ and fix an $L^{*}$-almost closed set $F \subset\left(L^{*} \backslash L\right) \cap(\leftarrow, x)_{L^{*}}$ such that $F \rightarrow x$. Using the fact that $\kappa$ is a regular cardinal it is easy to construct points $\left\{x_{\alpha}, y_{\alpha}, z_{\alpha}: \alpha<\kappa\right\}$ with the following properties:

(i) $x_{\alpha} \in A$ and $y_{\alpha}, z_{\alpha} \in F$ for each $\alpha<\kappa$;

(ii) $y_{\alpha}<x_{\alpha}<z_{\alpha}$ for every $\alpha<\kappa$;

(iii) if $\alpha<\beta<\kappa$, then $z_{\alpha}<y_{\beta}$;

(iv) the set $B=\left\{x_{\alpha}: \alpha<\kappa\right\}$ is cofinal in $(\leftarrow, x)_{L}$.

We claim that $B \subset A$ is an $L$-almost closed set with $B \rightarrow x$. The fact that $x \in \bar{B}$ is an immediate consequence of (iv), so let us establish that $B$ is almost closed. It is easy to see that every accumulation point $t<x$ of the set $B$ is the supremum of an infinite $B^{\prime} \subset B$. However, (ii) and (iii) imply that there is an infinite $F^{\prime} \subset F$ such that $\sup \left(F^{\prime}\right)=t$. The set $F \cap(\leftarrow, t]_{L^{*}}$ being closed, we have $t \in F \subset L^{*} \backslash L$ and therefore $t \notin L$. As a consequence, no point of $L \backslash(B \cup\{x\})$ is in the $L$-closure of $B$ so the set $B$ is almost closed and we proved that $L$ is a Whyburn space.

Now, if $L$ is a Whyburn space, suppose, for example, that $\chi\left(x,(\leftarrow, x]_{L}\right)>\omega$. Then $x \in \overline{(\leftarrow, x)_{L}}$ and therefore there is a discrete $D \subset(\leftarrow, x)_{L}$ with $x \in \bar{D}$ [DTTW Theorem 2.10]. Since $L$ is Whyburn, there exists an almost closed $B \subset D$ with $B \rightarrow x$. The set $F=\left(\operatorname{cl}_{L^{*}}(B) \backslash B\right) \cap(\leftarrow, x)_{L^{*}}$ is an $L^{*}$-almost closed subset of $\left(L^{*} \backslash L\right) \cap(\leftarrow, x)_{L^{*}}$ and $F \rightarrow x$. Hence $L$ is club incomplete. 
Recall that $X$ is a Lindelöf $\Sigma$-space if $X$ is a continuous image of a space $Y$ which can be perfectly mapped onto a second countable space.

2.11. Proposition. Let $L$ be a linearly ordered Lindelöf $\Sigma$-space. Then $L$ is Whyburn if and only if it is first countable.

Proof. Since tightness coincides with character in linearly ordered spaces (see En. 3.12.4(d)]), it suffices to prove that if $L$ has the Whyburn property, then $t(L) \leq \omega$. Suppose, then that $A \subset L$ and $x \in \bar{A}$. We can assume without loss of generality that $A \subset(\leftarrow, x)$ or $A \subset(x, \rightarrow)$; since these cases are identical we suppose that $A \subset(\leftarrow, x)$. There exists a discrete $D \subset A$ with $x \in \bar{D}$ [DTTW, Theorem 2.10]. Since $L$ is Whyburn, there exists an almost closed $B \subset D$ with $B \rightarrow x$. Observe that, for any open neighbourhood $U$ of the point $x$, the set $B \backslash U$ is closed and discrete in $L$; since $L$ is Lindelöf, $B \backslash U$ has to be countable. Furthermore $B \cup\{x\}$ is a Lindelöf $\Sigma$-space being closed in $L$. If $x \in \bar{C}$ for some countable $C \subset B$, then we are done. If not, then $B \cup\{x\}$ is homeomorphic to one-point Lindelöfication of an uncountable discrete space and hence it cannot be a Lindelöf $\Sigma$-space (see e.g. [Ar Proposition IV.6.15]). This contradiction shows that $t(L) \leq \omega$ and therefore $L$ is first countable.

The following example shows that Proposition 2.11 is not true if only Lindelöf property of $L$ is assumed.

2.12. Example. There exists a Lindelöf linearly ordered Whyburn space of uncountable tightness.

Proof. Let $M=\left(\omega_{1} \times[0,1)\right) \cup\left\{\omega_{1}\right\}$. The order is lexicographic and $\omega_{1}$ is the largest element of $M$. It is well-known that $M$ is compact (and hence complete) and $\omega_{1}$ is the unique point of $M$ of uncountable character. If $L=M \backslash\left(\omega_{1} \times\{0\}\right)$, then $M=L^{*}$ and $F=\omega_{1} \times\{0\}$ is an $L^{*}$-almost closed subset of $\left(L^{*} \backslash L\right) \cap\left(\leftarrow, \omega_{1}\right)_{M}$ with $F \rightarrow \omega_{1}$. Apply Theorem 2.10 to conclude that $L$ is a Whyburn space. Since the subspace $L \backslash U$ is second countable for each open $U \ni \omega_{1}$, the space $L$ is Lindelöf. Finally, if $A=\omega_{1} \times\{1 / 2\}$, then $\omega_{1} \in \bar{A}$ but no countable subset of $A$ contains $\omega_{1}$ in its closure.

\section{OpEN PROBLEMS}

The topics of this paper are still far from being exhausted. To illustrate this we present a list of open problems; their solutions will certainly require methods different from those developed in this paper.

3.1. Problem. Suppose that $G$ is a pseudocompact Whyburn group. Must $G$ be Fréchet-Urysohn? What if $G$ is separable?

3.2. Problem. Let $G$ be a (weakly) Whyburn group. Suppose that $H$ is a topological group and $f: G \rightarrow H$ is an open continuous surjective group homomorphism. Is $H$ necessarily a (weakly) Whyburn space?

3.3. Problem. Is it true that, under $\mathrm{MA}+\neg \mathrm{CH}$, any Hausdorff countably compact space of character $\leq \omega_{1}$ is weakly Whyburn? What if we omit "countably compact"?

3.4. Problem. Is it true that, under $\mathrm{MA}+\neg \mathrm{CH}$, any (Tychonoff) space of weight $\leq \omega_{1}$ is weakly Whyburn? 
3.5. Problem. Is it true that every (regular Lindelöf) $P$-space is weakly Whyburn?

3.6. Problem. Is it true that every (regular) $P$-space of cardinality $\leq \omega_{1}$ is a (weakly) Whyburn space?

3.7. Problem. For which compact spaces $X$ is the $\omega$-modification of $X$ weakly Whyburn? For example, is the $\omega$-modification of $\beta \omega \backslash \omega$ a weakly Whyburn space?

\section{REFERENCES}

[Ar] A.V. Arhangel'skii, Topological Function Spaces, Kluwer Academic Publishers, Mathematics and Its Applications 78, Dordrecht 1992. MR 92i:54022

[Be1] A. Bella, On spaces with the property of weak approximation by points, Comment. Math. Univ. Carolinae 35(1994), 357-360. MR 95e:54004

[Be2] A. Bella, Few remarks and questions on pseudoradial and related spaces, Topology Appl., 70(1996), 113-123. MR 97d:54048

[Bo] D. Booth, Ultrafilters on a countable set, Ann. Math. Logic 2:1(1970), 1-24. MR 43:3104

[BY] A. Bella and I.V. Yaschenko, On AP and WAP spaces, Comment. Math. Univ. Carolinae 40(1999), 531-536. MR 2001a:54006

[vD] E.K. van Douwen, The integers and topology, Handbook of Set-Theoretic Topology, ed. by K. Kunen and J.E. Vaughan, North Holland P.C., Amsterdam, 111-167. MR 87f:54008

[DTTW] A. Dow, M.G. Tkachenko, V.V.Tkachuk, R.G. Wilson, Topologies generated by discrete subspaces, Glasnik Matematicki, to appear.

[En] R. Engelking, General Topology, PWN, Warszawa, 1977. MR 58:18316b

[Ok] N.G. Okromeshko, Spaces onto which every quotient mapping is pseudo-open (in Russian), Comment. Math. Univ. Carolinae 23:1(1982), 1-10. MR 83g:54022

[PT] A. Pultr and A. Tozzi, Equationally closed subframes and representations of quotient spaces, Cahiers de Topologie et Geometrie Differentielle Categoriques 34(1993), 167183. MR 94f: 18003

[Si] P. Simon, On accumulation points, Cahiers de Topologie et Geometrie Differentielles Categoriques 35(1994), 321-327. MR 96a:54038

[TY] V.V. Tkachuk and I.V. Yaschenko, Almost closed sets and topologies they determine, Comment. Math. Univ. Carolinae 42:2(2001), 395-405. MR 2002b:54004

[Us] V.V. Uspenskij, On the spectrum of frequencies of function spaces (in Russian), Vestnik Mosk. Univ., Matem., Mech., 37:1(1982), 31-35.

[Wh] G.T. Whyburn, Accessibility spaces, Proceedings Amer. Math. Soc. 24(1970), 181-185. MR 40:1973

Institute of Mathematics, Čech Academy of Sciences, Žıtna 25, 11567, Prague, Čech REPUBLIC

E-mail address: pelant@math.cas.cz

Departamento de Matemáticas, Universidad Autónoma Metropolitana, Av. San Rafael Atlixco, 186, Col. Vicentina, Iztapalapa, C.P. 09340, México D.F.

E-mail address: mich@xanum.uam.mx

Departamento de Matemáticas, Universidad Autónoma Metropolitana, Av. San Rafael Atlixco, 186, Col. Vicentina Iztapalapa, C.P. 09340, México D.F.

E-mail address: vova@xanum.uam.mx

Departamento de Matemáticas, Universidad Autónoma Metropolitana, Av. San Rafael Atlixco, 186, Col. Vicentina Iztapalapa, C.P. 09340, México D.F.

E-mail address: rgw@xanum.uam.mx 